\title{
Near-field radiative heat transfer between general materials and metamaterials
}

\author{
ZHENG ZhiHeng \& XUAN YiMin* \\ School of Energy and Power Engineering, Nanjing University of Science and Technology, Nanjing 210094, China
}

Received February 25, 2011; accepted April 16, 2011

\begin{abstract}
We investigated the near-field radiative heat transfer between general materials and metamaterials. We studied the effects of metamaterial parameters on the radiative heat exchange and used three kinds of natural or artificially-constructed materials such as $\mathrm{Al}$, boron-doped $\mathrm{Si}$ and metamaterials as examples. We calculated and analyzed the near-field radiative heat transfer processes between two semi-infinite bodies. The numerical results indicate that the radiative heat exchange between the two different materials may be less or more than the radiative heat exchange between the corresponding identical materials. It was found out to depend on the radiative properties of the materials. The work would provide a valuable reference for the selection of practical materials.
\end{abstract}

near-field effect, near-field radiative heat transfer, material assembly, metamaterial

Citation: Zheng Z H, Xuan Y M. Near-field radiative heat transfer between general materials and metamaterials. Chinese Sci Bull, 2011, 56: 2312-2319, doi: $10.1007 / \mathrm{s} 11434-011-4586-9$

The swift advancement of micro/nanotechnology strongly encourages the development of highly-efficient miniature energy conversion and utilization devices [1-4] in which microscopic thermal transport is very complicated and needs to be considered [5]. Meanwhile, the appearance of a variety of man-made materials brings new challenges in near-field thermal radiation.

As an important part of the micro/nanoscale radiative heat transfer, various aspects of near-field radiative heat transfer has been studied by many scholars [6-18]. From their investigations we know that the macroscopic thermal radiation law fails to describe the radiative heat transfer processes at the subwavelength scale. Additionally, the near-field radiative heat transfer rate between two closely spaced bodies may increase by several orders of magnitude compared with that between two blackbodies at the macroscopic level because of near-field effects such as wave interference, photon tunneling and the excitation of surface waves [6-18]. Recently, the near-field radiative heat trans-

*Corresponding author (email: ymxuan@mail.njust.edu.cn) fer between two metamaterials was studied theoretically by Joulain et al. [19] and they found that the excitation of surface waves during TE polarization and the ferromagnetic behavior of the metamaterials will result in new channels for heat transfer and will enhance the radiative heat transfer. Therefore, the near-field radiative heat transfer becomes much more complicated because of the presence of metamataterials. However, some interesting questions arise such as the type of effects that may be induced by the parameters of metamaterials on the near-field radiative heat transfer, the results of the near-field radiative heat transfer between metamaterials and nonmagnetic materials (i.e. relative permeability $\mu=1$ ) and the difference in the near-field radiative heat transfer compared with that between the corresponding identical metamaterials or between the corresponding identical nonmagnetic materials. Wang et al. [12] analyzed the effects of material parameters as well as the temperature on the near-field heat transfer between the two identical normal materials using the Drude and Lorentz models. Fu and Zhang [20] comprehensively investigated the near-field radiative heat transfer between silicon surfaces with varying 
doping concentrations. Joulain et al. [19] studied the nearand far-field radiative heat transfer between two semi-infinite magnetodielectric materials by a combination of fluctuational electrodynamics and the fluctuation-dissipation theorem and found that the radiative heat transfer increases in the near field when surface waves are excited. Little attention has been paid to the near-field radiative heat transfer between two surfaces with dissimilar materials, especially between metamaterials and non-magnetic materials. A study into these properties will provide a valuable reference for the selection of material pairs in microscaled energy conversion devices such as thermophotovoltaic (TPV) systems.

We mainly investigated the radiative heat exchange between two semi-infinite bodies made of different materials including natural and artificial materials and we paid special attention to the effects of the metamaterial parameters on the near-field radiative heat exchange between two semiinfinite surfaces separated by a vacuum gap. The three types of materials used were $\mathrm{Al}$ [14,21], Si-19 (which refers to boron-doped $\mathrm{Si}$ at a concentration of $10^{19} \mathrm{~cm}^{-3}$ [12]) and metamaterials.

\section{Mathematical model}

The structural model is shown in Figure 1. We concentrated on the radiative heat flux between two semi-infinite bodies (media 1 and 3 ) separated by a vacuum gap (medium 2 ). Media 1 and 3 are maintained at temperatures $T_{1}$ and $T_{3}$, respectively. Because of the presence of fluctuating electrical and magnetic current sources in materials at temperatures higher than $0 \mathrm{~K}$, fluctuating electromagnetic fields are present inside as well as outside the material, and thermal radiation takes place [22]. The fluctuating electromagnetic field is calculated using fluctuating-dissipation theory [23] and Maxwell's stochastic equations, which is constructed by adding extra fluctuating electrical and magnetic current sources into Maxwell's equations [22]. The spectral heat flux transferred to medium 3 because of the fluctuating current and magnetic current sources in medium 1 can be divided into two parts: One is due to the electric current source in medium 1 and the other is due to the magnetic current source in medium 1 [24]. The following expression is obtained [24]:

$$
\begin{aligned}
& \operatorname{Re}\left\{\frac{\omega^{2} \mu_{1} \mu_{1}^{*} \mu_{0} \varepsilon_{0} \operatorname{Im}\left(\varepsilon_{1}\right)(\omega) \Theta\left(\omega, T_{1}\right)}{8 \pi^{2} \mu_{3}^{*}} \int_{0}^{+\infty} \mathrm{d} \beta \frac{\beta k_{o 3}^{*}}{\left|\gamma_{1}\right|^{2} \operatorname{Im}\left(\gamma_{1}\right)}\right. \\
& \left.\times\left\{\frac{\gamma_{3}^{*}\left|\tilde{A}_{3}^{e}\right|^{2}}{k_{o 3}^{*}}+\frac{\left|\tilde{C}_{3}^{e}\right|^{2} \gamma_{3}\left(\left|\gamma_{1}\right|^{2}+\beta^{2}\right)}{k_{o 3} k_{o 1} k_{o 1}^{*}}\right\} \mathrm{e}^{-2 \operatorname{Im}\left(\gamma_{2}\right) d}\right\}
\end{aligned}
$$

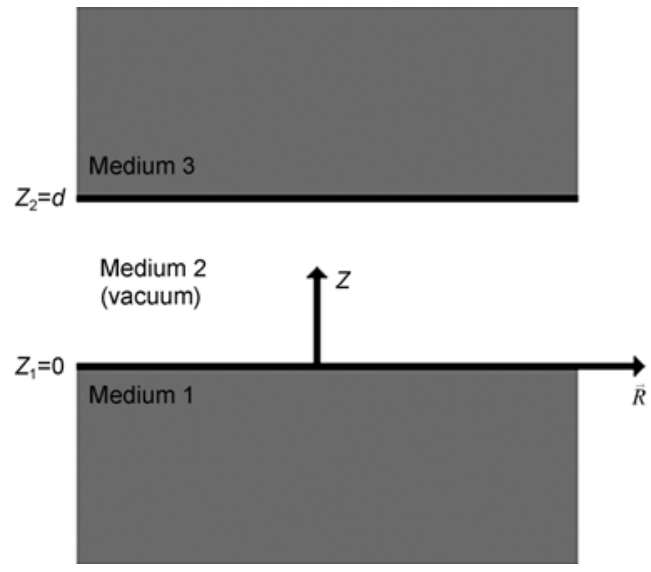

Figure 1 Schematic of the structure considered: the radiative heat flux is calculated between medium 1 and medium 3 with temperatures of $T_{1}$ and $T_{3}$, respectively. The vector $\vec{R}$ represents the direction $x \hat{x}+y \hat{y}$ where $\hat{x}$ and $\hat{y}$ respectively represent the unit vectors in the $x$ and $y$ directions.

$$
\begin{aligned}
& \operatorname{Re}\left\{\frac{\omega^{2} \varepsilon_{1} \varepsilon_{1}^{*} \varepsilon_{0} \mu_{0} \operatorname{Im}\left(\mu_{1}\right) \Theta\left(\omega, T_{1}\right)}{8 \pi^{2} \varepsilon_{3}} \int_{0}^{+\infty} \mathrm{d} \beta \frac{\beta k_{o 3}}{\left|\gamma_{1}\right|^{2} \operatorname{Im}\left(\gamma_{1}\right)}\right. \\
& \left.\times\left\{\frac{\gamma_{3}\left|\tilde{A}_{3}^{m}\right|^{2}}{k_{o 3}}+\frac{\left|\tilde{C}_{3}^{m}\right|^{2}\left(\gamma_{3}^{*}\left(\left|\gamma_{1}\right|^{2}+\beta^{2}\right)\right)}{k_{o 3}^{*} k_{o 1}^{*} k_{o 1}}\right\} \mathrm{e}^{-2 \operatorname{Im}\left(\gamma_{2}\right) d}\right\} .
\end{aligned}
$$

Eq. (1) shows the contribution of the electric current source in medium 1 and eq. (2) shows the contribution of the magnetic current source in medium 1 [24]. In eqs. (1) and (2), $\varepsilon_{0}$ and $\mu_{0}$ respectively stand for the permittivity and permeability of the vacuum while $\varepsilon$ and $\mu$ respectively are the relative electric permittivity and relative magnetic permeability. The subscripts 1 and 3 respectively represent media 1 and 3 and the superscript $*$ indicates a conjugated complex. Im and Re respectively represent the imaginary part and real component of the complex functions. $k_{o i}= \pm \sqrt{\omega^{2} \varepsilon_{0} \varepsilon_{i} \mu_{0} \mu_{i}}$ and $\gamma_{i}= \pm \sqrt{\omega^{2} \varepsilon_{0} \varepsilon_{i} \mu_{0} \mu_{i}-\beta^{2}}$, where $\beta$ is the parallel wave vector component. The signs for $k_{o i}$ and $\gamma_{i}$ follow those given in [25,26]. $\tilde{A}_{3}^{e}, \tilde{C}_{3}^{e}, \tilde{A}_{3}^{m}$ and $\tilde{C}_{3}^{m}$ are coefficients that can be determined by the transfer matrix method [27]. $\Theta\left(\omega, T_{1}\right)$ is the mean energy of a Planck oscillator at the frequency $\omega$. Details for the formulations can be found in [24]. A similar method can be applied to a calculation of the spectral heat flux that is transferred to medium 1 because of the fluctuating current and magnetic current sources in medium 3 . The difference between these two radiative heat fluxes is the net spectral radiative heat transfer between the two surfaces. The total net radiative heat transfer can be obtained by integrating over the frequency $\omega$ from zero to infinite. 


\section{Results and discussion}

\subsection{Relative electric permittivity and relative magnetic permeability models for metamaterials}

It is well known that metamaterials belong to a new class of electromagnetic composite materials and can exhibit a host of novel properties such as negative relative dielectric permittivity $(\varepsilon)$, negative relative magnetic permeability $(\mu)$ and a negative refraction index [28-32]. The metamaterial is both dispersive and dissipative and generally its relative dielectric and magnetic is complex and dependent on frequency $[19,33,34]$. For metamaterials made from an artificial array of wires and a split ring the effective relative permittivity and permeability can be expressed as $[19,30,31$, $33,35,36]$

$$
\begin{gathered}
\varepsilon(\omega)=1-\frac{\omega_{p}^{2}}{\omega^{2}+i \gamma_{e} \omega}, \\
\mu(\omega)=1-\frac{F \omega^{2}}{\omega^{2}-\omega_{o}^{2}+i \gamma_{m} \omega},
\end{gathered}
$$

where $F$ is the area proportionately occupied by the split ring in the unit cell, $\omega_{p}$ is the effective plasma frequency, $\omega_{o}$ is the resonance frequency, and $\gamma_{e}$ and $\gamma_{m}$ are the scattering rates $[19,35,36]$. By algebraic manipulations the real and imaginary parts of the relative permeability $\left(\mu=\mu^{\prime}+i \mu^{\prime \prime}\right)$ can be respectively expressed as

$$
\begin{gathered}
\mu^{\prime}=1-\frac{F \omega^{2}\left(\omega^{2}-\omega_{o}^{2}\right)}{\left(\omega^{2}-\omega_{o}^{2}\right)^{2}+\gamma_{m}^{2} \omega^{2}}, \\
\mu^{\prime \prime}=\frac{F \omega^{3} \gamma_{m}}{\left(\omega^{2}-\omega_{o}^{2}\right)^{2}+\gamma_{m}^{2} \omega^{2}} .
\end{gathered}
$$

Therefore, $\mu^{\prime \prime}$ is always greater than 0 for $\omega>0$ and is equal to 0 when $\omega=0$. By setting $\mu^{\prime}<0$, one can get

$$
(1-F) \omega^{4}+\left[(F-2) \omega_{o}^{2}+\gamma_{m}^{2}\right] \omega^{2}+\omega_{o}^{4}<0 .
$$

From eqs. (5a) and (6) it is clear that $\mu^{\prime}<0$ occurs in a frequency band above $\omega_{o}$ or it does not occur, which is determined by the parameters $F, \gamma_{m}$ and $\omega_{o}$. Since eq. (3) is similar to the Drude model, a detailed analysis of the influence of $\gamma_{e}$ on $\varepsilon$ has been given in $[12,13]$.

Figure 2 shows the influence of $\gamma_{m}$ and $\gamma_{e}$ on the nearfield radiative heat flux between two identical semi-infinite metamaterial bodies with a vacuum gap $d=10 \mathrm{~nm}$ for the parameters $\omega_{p}=10^{14} \mathrm{rad} / \mathrm{s}, \omega_{o}=0.4 \omega_{p}$, and $F=0.56$. Figure 2(a) corresponds to the influence of $\gamma_{e}$ on the body with $\gamma_{m}=0.01 \omega_{p}$ and Figure 2(b) corresponds to the influence of $\gamma_{m}$ on the body with $\gamma_{e}=0.01 \omega_{p}$. The numerical calculation was conducted under the condition that the temperatures of
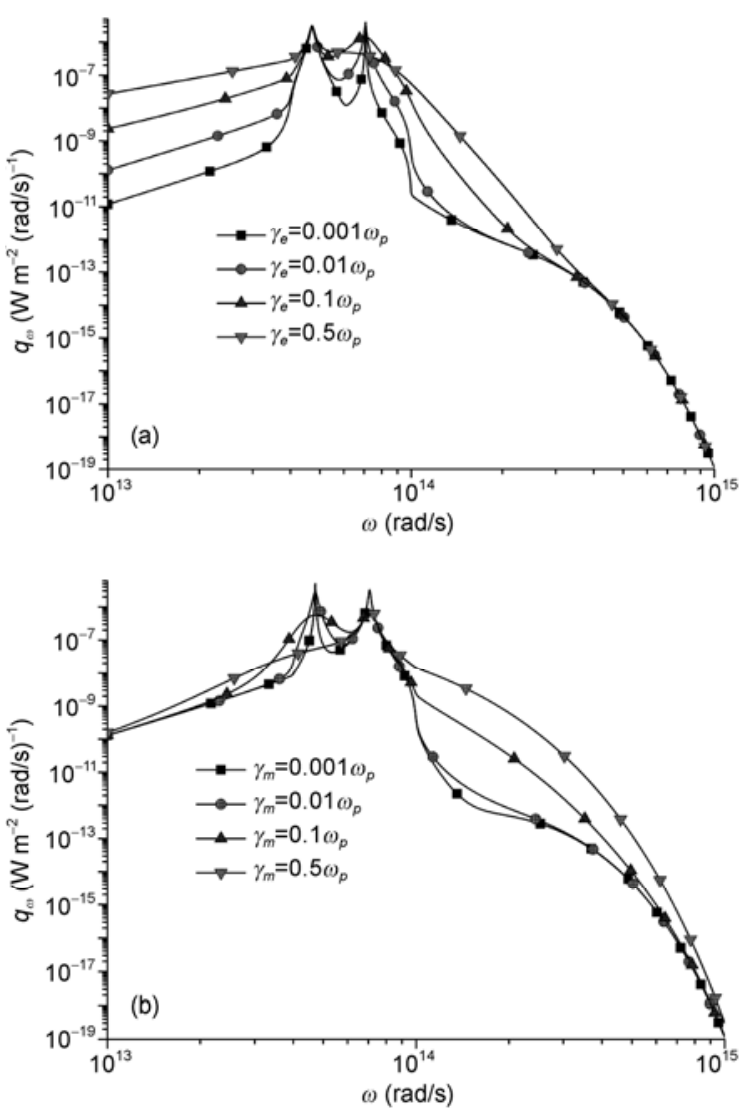

Figure 2 The effects of scattering rate on the spectral heat flux between two identical semi-infinite metamaterial bodies at the gap $d=10 \mathrm{~nm}$ with $\omega_{p}=10^{14}$ $\mathrm{rad} / \mathrm{s}, \omega_{o}=0.4 \omega_{p}$ and $F=0.56$. (a) The effect of $\gamma_{e}$; (b) the effect of $\gamma_{m}$.

the two surfaces were maintained at $T_{1}=300 \mathrm{~K}$ and $T_{3}=0 \mathrm{~K}$, respectively. As shown in Figure 2, the first peak at lower frequency $\omega=4.717 \times 10^{13} \mathrm{rad} / \mathrm{s}$ comes from the excitation of the surface waves in TE polarization and in a narrow frequency band around $\omega=4.717 \times 10^{13} \mathrm{rad} / \mathrm{s}$ while there is a large number of electromagnetic states, which results in a high amount of radiative heat transfer and is referred to as the resonance of the flux $[19,24,37]$. Similarly, the second peak at higher frequency $\omega=7.075 \times 10^{13} \mathrm{rad} / \mathrm{s}$ is due to the excitation of surface waves during TM polarization and the resonance of the flux $[19,24,37]$. It should be noted that since $\mu^{\prime}<0$ does not occur for $\gamma_{m}=0.5 \omega_{p}$ the dispersion relationship for the excitation of surface waves in TE polarization (i.e. $\mu_{2} \gamma_{1}+\mu_{1} \gamma_{2}=0$ ) cannot be satisfied. Therefore, no peak is present as a result of the excitation of surface waves in TE polarization for the curve corresponding to $\gamma_{m}=0.5 \omega_{p}$ in Figure 2(b). Furthermore, Figure 2 reveals that the frequencies of the peaks because of the excitation of surface waves during TE polarization in the spectral heat flux remain at the same locations. Similarly, the frequencies of the peaks that are due to the excitation of surface waves during TM polarization in the spectral heat flux are at the same locations. Both the parameters, $\gamma_{m}$ and $\gamma_{e}$, have little influ- 
ence on the locations of the peaks. The parameter $\gamma_{e}$ mainly determines the sharpness of the peak of heat flux because of the excitation of surface waves in TM polarization and this has little effect on the sharpness of the heat flux peak because of the excitation of surface waves during TE polarization. On the contrary, the parameter $\gamma_{m}$ mainly determines the sharpness of the heat flux peak because of the excitation of surface waves in TE polarization and this has little effect on the sharpness of the heat flux peak because of the excitation of surface waves during TM polarization. For a small $\gamma_{e}$ the corresponding heat flux peak is very sharp, which is similar to that obtained in [12]. For a small $\gamma_{m}$ the corresponding heat flux peak is also very sharp.

\subsection{Near-field radiative heat transfer between two surfaces with a different materials assembly}

The near-field radiative heat transfer between the two semiinfinite surfaces mode of a dissimilar materials assembly is more complicated than that discussed in the preceding section and they may exhibit quite different features. The presence of metamaterials further complicates the near-field radiative heat transfer. This section is devoted to a detailed analysis of the near-field radiative heat transfer between two semi-infinite bodies made of different materials. In the subsequent calculation the temperatures of the two semi-infinite bodies are respectively maintained at $T_{1}=300 \mathrm{~K}$ and $T_{3}=275 \mathrm{~K}$. Although one may exchange the materials of medium 1 and medium 3 , the expression for the net heat flux from medium 1 to medium 3 is formally identical, which has been shown in [19].

Figure 3 shows the spectral net radiative heat flux between the two semi-infinite bodies with the vacuum gap $d=1 \mu \mathrm{m}$ for several different combinations of metamaterials and $\mathrm{Si}-19$. Note that since the vacuum gap $d=1 \mu \mathrm{m}$ is comparable to the characteristic wavelength of thermal radiation for the given temperatures the near-field effect on the radiative heat transfer between the two surfaces can be clearly identified. Meanwhile, the vacuum gap $d=1 \mu \mathrm{m}$ is not too much smaller than the characteristic wavelength for the given temperatures. On the other hand, the far-field contribution may be considered as well. Therefore, the radiative heat exchange phenomena at the vacuum gap $d=1 \mu \mathrm{m}$ may be more complicated than that for a much smaller vacuum gap such as a nanometer gap where the near-field effect is dominant in the radiative heat flux. The selected parameters for the metamaterial as described by eqs. (3) and (4) are $\omega_{p}=10^{14} \mathrm{rad} / \mathrm{s}, \omega_{o}=0.4 \omega_{p}, \gamma_{e}=\gamma_{m}=0.012 \omega_{p}$ and $F=0.56$. The selection of these parameters for the metamaterial should match the given temperature range of media 1 and 3, which does not rely on the state of the art technology limitation. The parameters of the non-magnetic material $\mathrm{Si}-19$ are obtained from the Drude model $\varepsilon(\omega)=\varepsilon_{\infty}-\frac{\omega_{p}^{2}}{\omega^{2}+i \gamma \omega}$ with $\varepsilon(\infty)=11.7, \omega_{p}=3.42 \times 10^{14} \mathrm{rad} / \mathrm{s}$ and $\gamma=6.12 \times 10^{12} \mathrm{rad} / \mathrm{s}$ [12]. Note that to highlight the effects of the surface phonon polariton resonance, the value of parameter $\gamma=6.12 \times 10^{12} \mathrm{rad} / \mathrm{s}$ is taken to be one tenth that in [12]. For simplicity, the material described by these parameters is still referred to as Si-19 in the following text. For the curve corresponding to case 1 (as shown in Figure 3), three peaks are present that correspond to the frequencies $\omega_{1}=4.01 \times 10^{13} \mathrm{rad} / \mathrm{s}$, $\omega_{2}=4.71 \times 10^{13} \mathrm{rad} / \mathrm{s}$ and $\omega_{3}=7.707 \times 10^{13} \mathrm{rad} / \mathrm{s}$, respectively. The radiative heat transfer mainly increases because of the excitation of surface waves in TE polarization around $\omega_{2}$ and the excitation of surface waves in TM polarization around $\omega_{3}[19,24]$. The tiny peak at $\omega_{1}$ is due to the asymptotic behavior in TM polarization around $\omega=\omega_{0}$ where the relative permeability is very large [19,24]. For the curve that corresponds to case 3 , the radiative heat transfer is greatly enhanced because of the excitation of surface waves during TM polarization around $\omega=9.58 \times 10^{13} \mathrm{rad} / \mathrm{s}$ [12]. For the curve that corresponds to case 2 all the properties are retained for the two cases discussed above but the increases that result from these properties all decrease. The main reason is that the dissimilar materials cause the frequency band of the high radiation emission to be different from those of high absorption (i.e. $\left.\operatorname{Im}\left(r_{21}^{s, p}\right) \neq \operatorname{Im}\left(r_{23}^{s, p}\right)\right)$ [37,38]. Note that the excitation of the surface waves for TE polarization only occurs at the metamaterials/vacuum interface for case 2 while excitation occurs at both the metamaterials/vacuum interfaces for case 1 and the two surface waves get coupled [24]. As shown in Table 1, it is also noteworthy that the total heat flux for case 2 is much lower than that for the other two cases.

To further understand the effect of surface waves on the radiative heat transfer between the two semi-infinite bodies, the radiative heat flux can be plotted as a function of frequency $\omega$ and the parallel wave vector component $\beta$. As shown in Figure 4, the angular frequency $\omega$ is normalized to

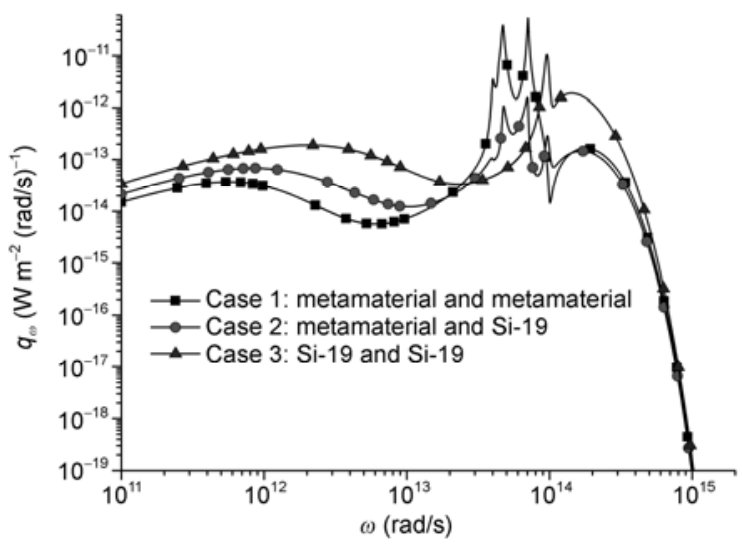

Figure 3 The spectral net radiative heat flux between the two semi-infinite bodies with the gap $d=1 \mu \mathrm{m}$ for different assemblies of the metamaterial and $\mathrm{Si}-19$. 
Table 1 Comparison of the radiative heat flux among the different material assemblies and gaps

\begin{tabular}{lcc}
\hline \multicolumn{1}{c}{ Material assembly } & Heat flux at $d=1 \mu \mathrm{m}\left(\mathrm{W} / \mathrm{m}^{2}\right)$ & Heat flux at $d=10 \mathrm{~nm}\left(\mathrm{~W} / \mathrm{m}^{2}\right)$ \\
\hline Metamaterial and metamaterial & 396.377 & 3493366.822 \\
Metamaterial and Si-19 & 46.870 & 165643.529 \\
Metamaterial and Al & 6.850 & 127615.333 \\
Si-19 and Si-19 & 325.423 & 875797.090 \\
Si-19 and Al & 5.819 & 385.492 \\
Al and Al & 4.215 & 111923.507 \\
\hline
\end{tabular}
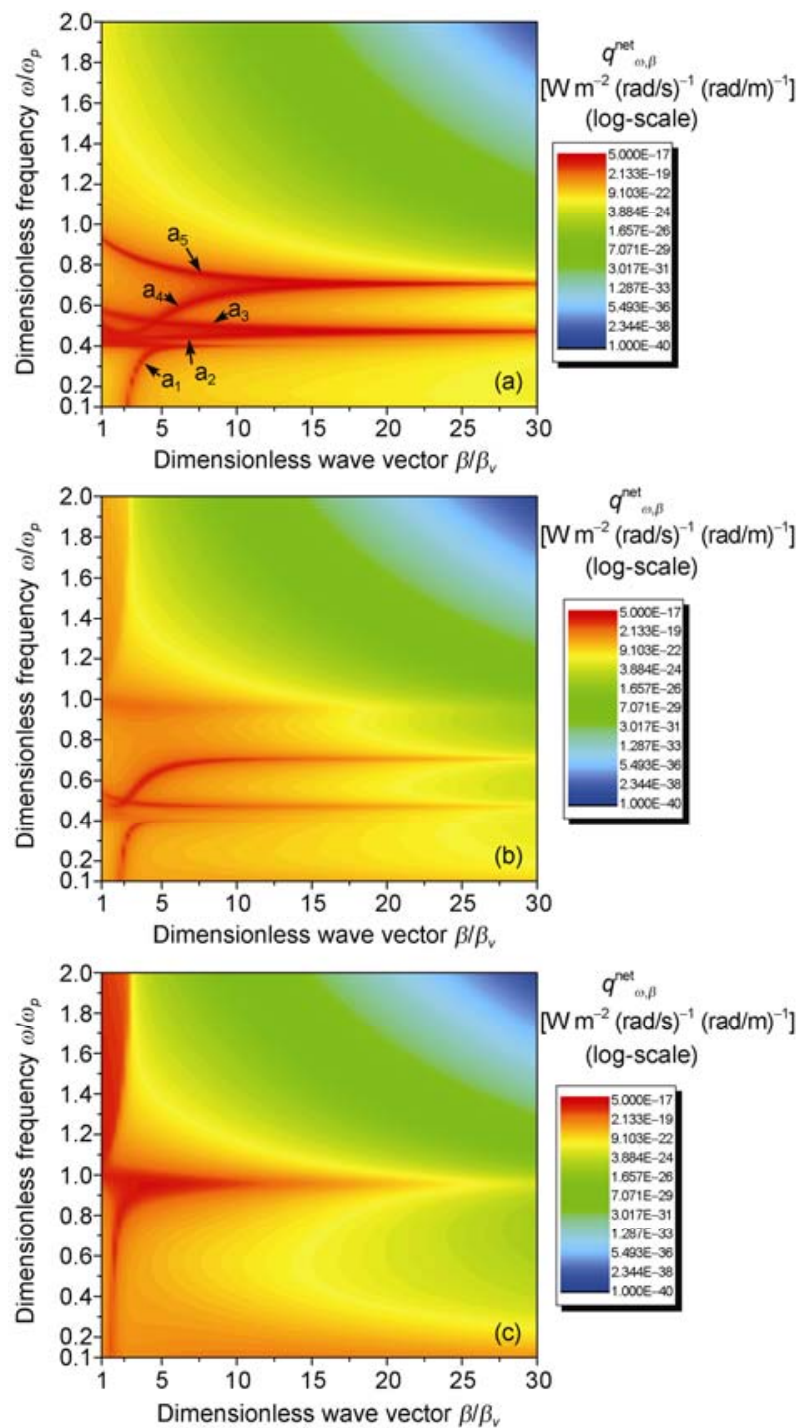

Figure 4 Spectral distribution of the net radiative heat flux (in log-scale) from medium 1 to medium 3 as a function of the parallel wave vector component $\beta$ and the frequency $\omega$ at a gap of $1 \mu \mathrm{m}$. (a) The assembly of the metamaterial and metamaterial; (b) the assembly of the metamaterial for medium 1 and Si-19 for medium 3; (c) the assembly of Si-19 and Si-19.

the effect plasma frequency $\omega_{p}=1 \times 10^{14} \mathrm{rad} / \mathrm{s}$ and the parallel wave vector component $\beta$ is normalized with respect to the wave vector in vacuum $\beta_{v}$, i.e. $\beta_{v}=\omega / c$. Figures $4(\mathrm{a})-(\mathrm{c})$ correspond to cases 1-3, respectively, in Figure 3. Five dispersion curves are present in Figure 4(a). Curves $a_{2}$ and $a_{3}$ represent the effects of the excitation of surface waves during TE polarization. Curves $a_{1}, a_{4}$ and $a_{5}$ represent the effects of the excitation of surface waves in TM polarization. Curve $\mathrm{a}_{1}$ becomes flatter as $\beta$ increases because of the asymptotic behavior at $\omega=\omega_{o}$ resulting in a tiny heat flux peak for case 1 in Figure $3[19,24]$. The radiative heat transfer is greatly enhanced by the excitation of surface waves during the TE and TM polarizations. As shown in Figure 4(b), if one of the semi-infinite bodies is made of Si-19 instead of the metamaterial two curves, $a_{2}$ and $a_{5}$, appear in Figure 4(a) and disappear in Figure 4(b) because of the alteration of the surface waves excitation as a result of the dissimilar material assembly. Additionally, the intensities of the radiative heat transfer for curves $a_{1}, a_{3}$ and $a_{4}$ as shown in Figure 4(b) are greatly diminished, which results in a larger decrease in the radiative heat exchange between the two surfaces.

Although the radiative heat transfer is enhanced by the excitation of surface waves during TM polarization because of the presence of $\mathrm{Si}-19$, such an increase is not large enough to balance these decreases because of the dissimilar material assembly. Therefore, in Figure 3 the total radiative heat exchange that corresponds to case 2 is much smaller than that for case 1. As shown in Figure 4(c), for the case where both surfaces are made of $\mathrm{Si}-19$, the radiative heat transfer is greatly enhanced by the excitation of the surface waves of Si-19 during TM polarization where the frequency is less than $9.97 \times 10^{13} \mathrm{rad} / \mathrm{s}$. Furthermore, one can also see a remarkable enhancement at higher than $9.97 \times 10^{13} \mathrm{rad} / \mathrm{s}$, which is due to the higher $\operatorname{Im}\left(r_{21}^{s}\right)$ and $\operatorname{Im}\left(r_{21}^{p}\right)$ values in these ranges. By a comparison of the curves in Figure 4(b) and (c), a curve that corresponds to the excitation of surface waves in TM polarization in Figure 4(c) at less than $9.58 \times 10^{13} \mathrm{rad} / \mathrm{s}$ vanishes in Figure 4(b). Additionally, the intensities of the increase in radiative heat transfer because of the surface waves during TM polarization and because of the higher values of $\operatorname{Im}\left(r_{21}^{s}\right)$ and $\operatorname{Im}\left(r_{21}^{p}\right)$ at higher than $9.58 \times 10^{13} \mathrm{rad} / \mathrm{s}$ in Figure 4(c) are greatly diminished in Figure 4(b), which results in a greater decrease in radiative heat transfer for the dissimilar material assembly. On the other hand, although the radiative heat transfer is also en- 
hanced by the excitation of surface waves during TE- and TM-polarization because of the presence of metamaterials, such an increase is not large enough to balance the decrease that results from the dissimilar material assembly. Therefore, the total heat exchange for case 2 in Figure 3 is much lower than that for the other two cases that correspond to the same material assembly.

To estimate the near-field effect, a further calculation was carried out for the situation where the vacuum gap between the two surfaces was $10 \mathrm{~nm}$ for the above-mentioned three material assemblies. As shown in Figure 5, with a decrease in the width of the vacuum gap, the near-field effect becomes stronger and more remarkable so that the radiative heat exchange between the two surfaces is dominated by evanescent waves (i.e. photon tunneling) [39]. A comparison between the curves in Figure 5 and those in Figure 3 reveals that the total radiative heat exchange flux for the three types of material assemblies for the $10 \mathrm{~nm}$ gap is much larger than that of the corresponding $1 \mu \mathrm{m}$ gap, as shown in Table 1 . The data listed in Table 1 more clearly explains the near-field effect on the radiative heat transfer between the two semi-infinite surfaces. Because of the strong near-field effect at the $10 \mathrm{~nm}$ gap some of the phenomena observed in Figure 3 become submerged and some peaks in the curves in Figure 3 disappear in Figure 5. The assembly of the metamaterials and Si-19 may be used as an example to further explain the near-field effect. As shown in Figure 6 , the increase because of the excitation of the surface waves during TE polarization is smaller because of the excitation of the surface waves during TM polarization at the matematerial/vacuum interface when the gap was $10 \mathrm{~nm}$. This was not observed in Figure 5 although it does exist, which means that for this different materials assembly with a $10 \mathrm{~nm}$ gap the TM-wave contribution is a dominant factor for the radiative heat transfer flux. Additionally, the enhancement because of the asymptotic behavior during TM polarization at $\omega=\omega_{o}$ becomes negligible for both the assembly

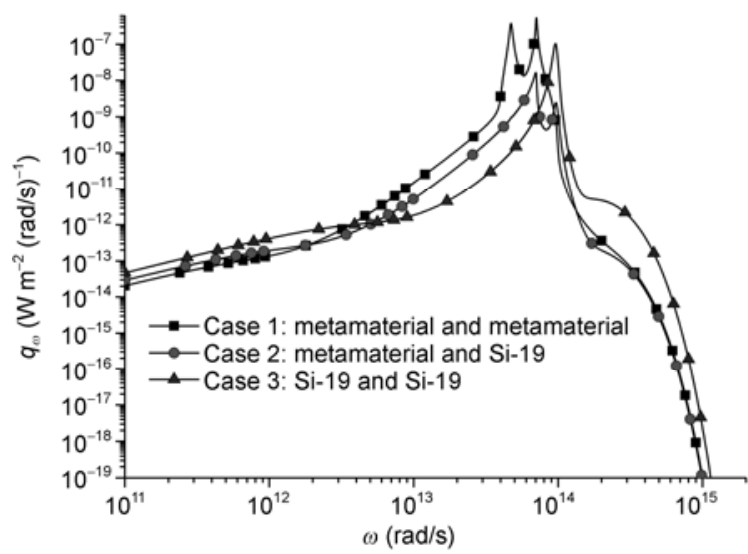

Figure 5 The spectral net radiative heat flux between the two semi-infinite bodies with the gap $d=10 \mathrm{~nm}$ for the different assemblies between the metamaterial and Si-19.

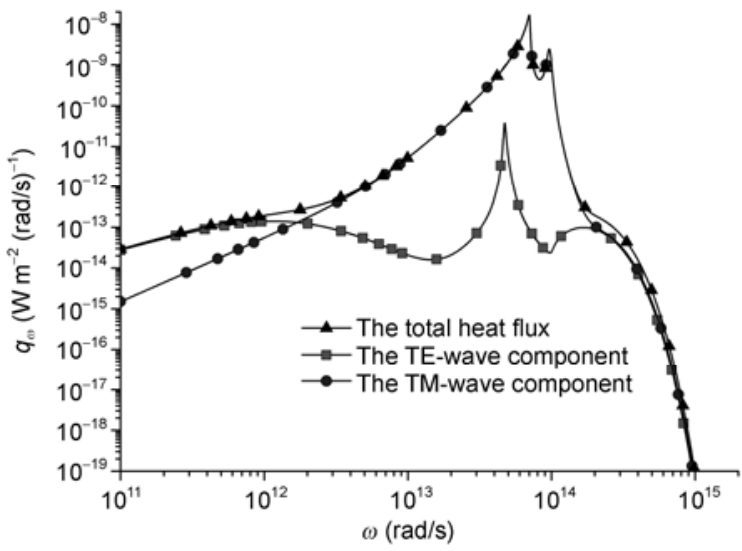

Figure 6 The TE- and TM-wave contributions to the spectral radiative flux and the total spectral radiative flux where the metamaterial is for medium 1 and Si-19 for medium 3 with the gap $d=10 \mathrm{~nm}$.

cases wherein the metamaterial is involved if the vacuum gap is $10 \mathrm{~nm}$ since the corresponding tiny peaks cannot be found in the corresponding curves in Figure 5 [19,24].

Figure 7 reveals the spectral net radiative heat flux between two semi-infinite bodies with the vacuum gap $d=1 \mu \mathrm{m}$ for the three different combinations of metamaterial and $\mathrm{Al}$. The parameters for $\mathrm{Al}$ is described by the Drude model $\varepsilon(\omega)=\varepsilon_{\infty}-\frac{\omega_{p}^{2}}{\omega^{2}+i \gamma \omega}$ with $\omega_{\infty}=1, \omega_{p}=2.4 \times 10^{16} \mathrm{rad} / \mathrm{s}$ and $\gamma=1.25 \times 10^{14} \mathrm{rad} / \mathrm{s}[14,21]$ while the parameters for the metamaterials is identical to that in Figure 3. In Figure 7, case 1 was discussed above. Case 2 is where medium 1 is the metamaterial and medium 3 is Al. In this case, the effects of the excitation of the surface waves of the metamaterial during TE polarization on heat transfer are preserved but greatly diminished and during TM polarization they are almost eliminated, as shown in Figure 8. Therefore, the spectral heat flux is drastically reduced at frequencies higher than $4.71 \times 10^{13} \mathrm{rad} / \mathrm{s}$ for case 2 in Figure 7 . Note that

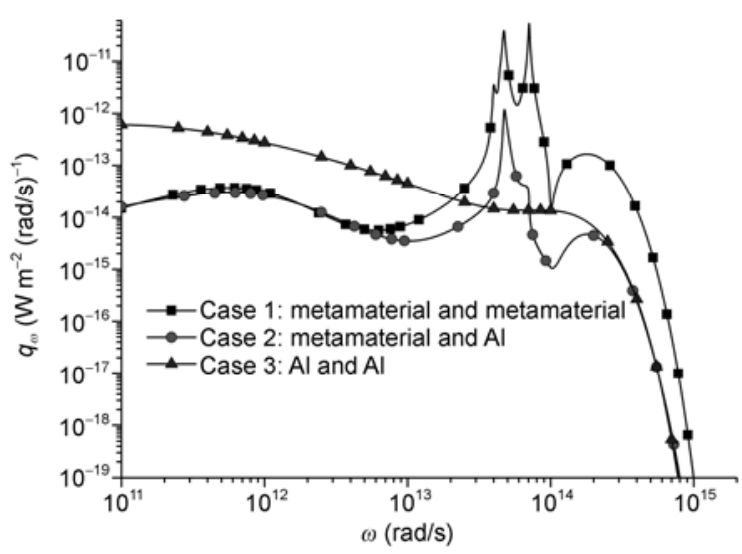

Figure 7 The spectral net radiative heat flux between two semi-infinite bodies with the gap $d=1 \mu \mathrm{m}$ for different assemblies of the metamaterial and $\mathrm{Al}$. 


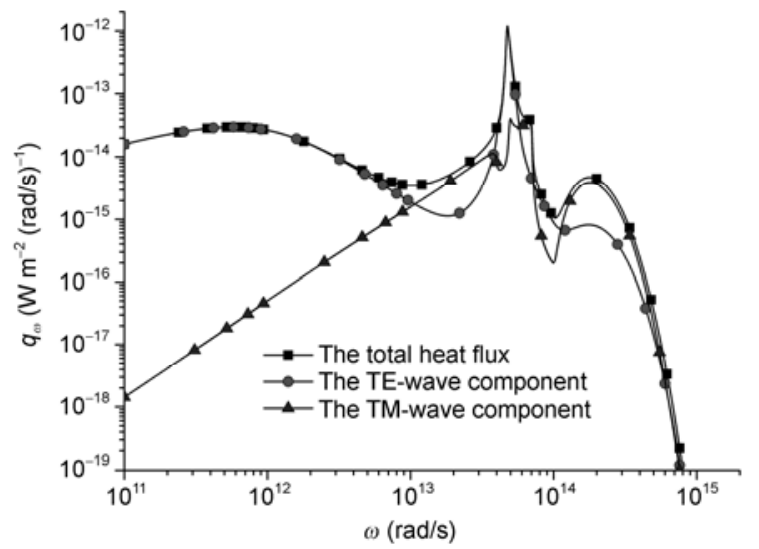

Figure 8 The TE- and TM-wave contributions to the spectral radiative flux and the total spectral radiative flux where the metamaterial is for medium 1 and $\mathrm{Al}$ for medium 3 with the gap $d=1 \mu \mathrm{m}$.

although surface plasmon polaritons also possibly excite at the $\mathrm{Al} /$ vacuum interface, the excitation frequency is not in the frequency domain where the contribution to the heat flux is significant for the given temperature and it has little influence on the radiative heat transfer [14]. It is emphasized that the radiative heat transfer for the metamaterialmetamaterial assembly is the highest among all three types of material assemblies. Further calculations for the three possible assemblies of $\mathrm{Al}$ and metamaterial at the $10 \mathrm{~nm}$ gap was carried out to understand the near-field effect, as illustrated in Figure 9. A similar phenomenon to that observed for the same material assembly at the $1 \mu \mathrm{m}$ gap was obtained but the near-field effect becomes more evident. The effect of the excitation of surface polaritons during TM polarization was not observed for case 2 . Note that in cases 2 and 3, both the radiative heat transfers are dominated by the TE-wave contribution. The presence of Al drastically reduces the TM-wave contribution to the radiative heat flux. The material assembly of $\mathrm{Al}$ and the metamaterial leads to quite a different radiative heat transfer phenomenon from

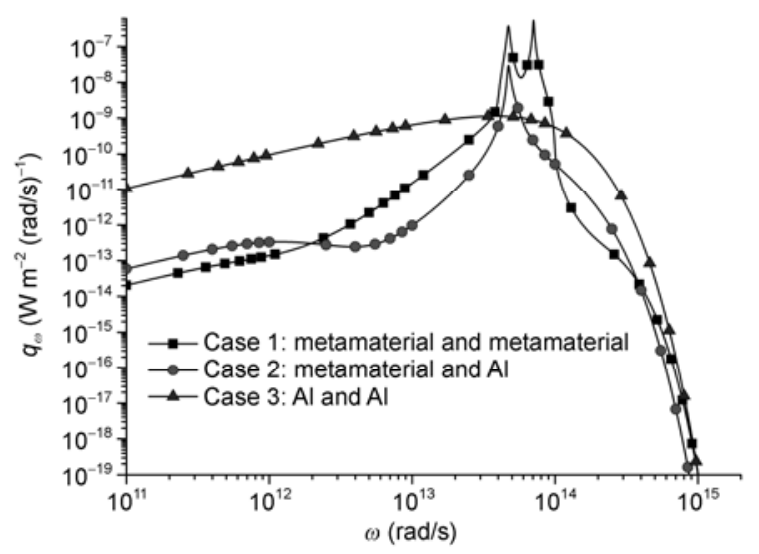

Figure 9 The spectral net radiative heat flux between the two semi-infinite bodies with the vacuum gap $d=10 \mathrm{~nm}$ for the different assemblies of metamaterials and $\mathrm{Al}$. that observed for the above-mentioned assemblies of Si-19 and the metamaterial. The radiative heat transfer for the former is dominated by a TE-wave contribution and that for the latter is dominated by a TM-wave contribution.

We will now discuss the near-field radiative heat transfer for the assembly of $\mathrm{Al}$ and $\mathrm{Si}-19$. Figure 10 shows the spectral net radiative heat flux between the two semi-infinite bodies with the vacuum gap $d=10 \mathrm{~nm}$ for the three different combinations of $\mathrm{Si}-19$ and $\mathrm{Al}$. It is clear that the radiative heat exchange for case 2 is much smaller than that for cases 1 and 3 . The total radiative heat flux for cases 1 and 3 are several orders of magnitude higher than that for case 2 . The main reason is that the effects of the excitation of surface polaritons of Si-19 during TM polarization decreases greatly because of the presence of $\mathrm{Al}$ and there are no other effects of the excitation of surface polaritons during TE or TM polarization to enhance the radiative heat transfer in the thermal frequency band for case 2 .

\section{Conclusions}

We investigated the near-field radiative heat transfer between two semi-infinite surfaces made from different materials assemblies of $\mathrm{Al}$, boron-doped $\mathrm{Si}$ and metamaterial. The effects of the material parameters $\gamma_{e}$ and $\gamma_{m}$ on the radiative heat transfer are discussed. The results show that both the parameters $\gamma_{e}$ and $\gamma_{m}$ have little influence on the locations of the peaks. They mainly determine the sharpness of the heat flux peak because of the excitation of surface waves during TM polarization and TE polarization, respectively.

Among all the material assemblies, the radiative heat transfer for the assembly of the metamaterial-metamaterial is the highest. Compared with the radiative heat transfer of two surfaces made from the same material assembly, the radiative heat exchange between two dissimilar materials can be decreased or increased, which is dependent on the

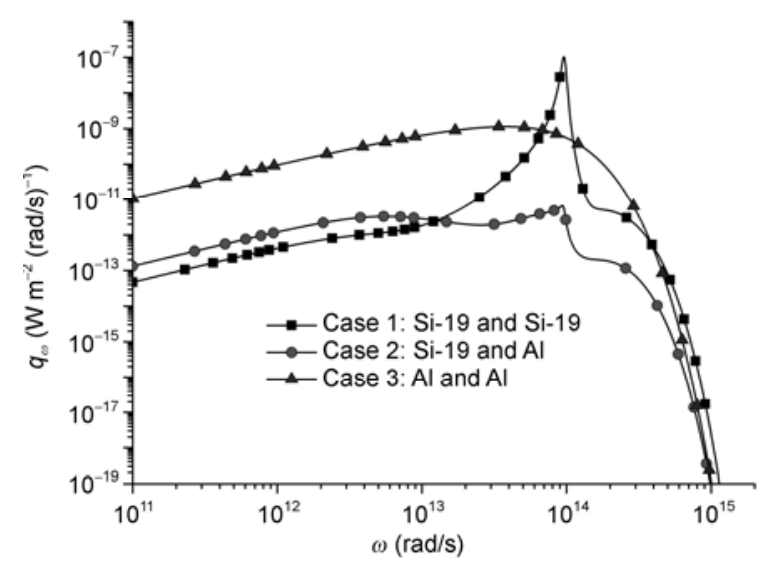

Figure 10 The spectral net radiative heat flux between two semi-infinite bodies with the gap $d=10 \mathrm{~nm}$ for different assemblies of Si-19 and Al. 
material characteristics. If the material assembly is the metamaterial and $\mathrm{Si}-19$, the mismatch between the spectral bands of high radiation emission and high absorptance may drastically reduce the radiative heat flux and the radiative heat transfer and it is much smaller than that for the same material assembly of the metamaterials or Si-19.

The presence of $\mathrm{Al}$ in a material assembly can drastically reduce the TM-wave contribution to the radiative heat flux. Therefore, the radiative heat transfer between the metamaterial and $\mathrm{Al}$ is much smaller than that between the two metamaterials because of the intensive reduction of the TM contribution. The radiative heat transfer between $\mathrm{Al}$ and the non-magnetic material $\mathrm{Si}-19$ is also much smaller than that between the identical $\mathrm{Si}-19$ because the assembly of $\mathrm{Al}$ and Si-19 suppresses the excitation of the surface polaritons in TM polarization.

This work was supported by the National Natural Science Foundation of China (50936002).

1 Chowdhury I, Prasher R, Lofgreen K, et al. On-chip cooling by superlattice-based thin-film thermoelectrics. Nat Nanotechnol, 2009, 4: 235-238

2 Schwede J W, Bargatin I, Riley D C, et al. Photon-enhanced thermionic emission for solar concentrator systems. Nat Mater, 2010, 9: 762-767

3 Basu S, Chen Y B, Zhang Z M. Microscale radiation in thermophotovoltaic devices - A review. Int J Energy Res, 2007, 31: 689-716

4 Schubert E F. Light-emitting Diodes. 2nd ed. Cambridge: Cambridge University Press, 2006

5 Shakouri A. Nanoscale thermal transport and microrefrigerators on a chip. Proc IEEE, 2006, 94: 1613-1638

6 Pendry J B. Radiative exchange of heat between nanostructures. J Phys: Condens Matter, 1999, 11: 6621-6633

7 Joulain K, Mulet J-P, Marquier F, et al. Surface electromagnetic waves thermally excited: radiative heat transfer, coherence properties and Casimir forces revisited in the near field. Surf Sci Rep, 2005, 57 : 59-112

8 Basu S, Zhang Z M. Maximum energy transfer in near-field thermal radiation at nanometer distances. J Appl Phys, 2009, 105: 093535

9 Francoeur M, Menguc M P, Vaillon R. Solution of near-field thermal radiation in one-dimensional layered media using dyadic Green's functions and the scattering matrix method. J Quant Spectrosc Radiat Transfer, 2009, 110: 2002-2018

10 Narayanaswamy A, Chen G. Direct computation of thermal emission from nanostructures. Ann Rev Heat Transfer, 2005, 14: 169-195

11 Volokitin A, Persson B N J. Near-field radiative heat transfer and noncontact friction. Rev Mod Phys, 2007, 79: 1291-1329

12 Wang X J, Basu S, Zhang Z M. Parametric optimization of dielectric functions for maximizing nanoscale radiative transfer. J Phys D: Appl Phys, 2009, 43: 245403

13 Basu S, Zhang Z M, Fu C J. Review of near-field thermal radiation and its application to energy conversion. Int J Energy Res, 2009, 33: 1203-1232

14 Fu C J, Tan W C. Near-field radiative heat transfer between two plane surfaces with one having a dielectric coating. J Quant Spectrosc Radiat Transfer, 2009, 110: 1027-1036

15 Mulet J-P, Joulian K, Carminati $\mathrm{R}$, et al. Enhanced radiative heat transfer at nanometric distances. Microscale Thermophys Eng, 2002, 6: 209-222

16 Polder D, Van Hove M. Theory of radiative heat transfer between closely spaced bodies. Phys Rev B, 1971, 4: 3303-3314

17 Shuai Y, Che Z Z, Zhang H C, et al. Monochromatic effect and polarization in near thermal field radiation of surfaces. J Eng Thermophys, 2008, 29: 1002-1004

18 Han M H, Liang X G. Study on near-field radiative heat transfer of spherical particles. J Eng Thermophys, 2007, 28: 107-109

19 Joulain K, Drevillon J, Ben-Abdallah P. Noncontact heat transfer between two metamaterials. Phys Rev B, 2010, 81: 165119

20 Fu C J, Zhang Z M. Nanoscale radiation heat transfer for silicon at different doping levels. Int $\mathbf{J}$ Heat Mass Transfer, 2006, 49: 1703-1718

21 Biehs S-A, Reddig D, Holthaus M. Thermal radiation and near-field energy density of thin metallic films. Eur Phys J B, 2007, 55: 237-251

22 Loomis J J, Maris H J. Theory of heat transfer by evanescent electromagnetic waves. Phys Rev B, 1994, 50: 18517-18524

23 Rytov S M, Kravtsov Yu A, Tatarskii V I. Principles of Statistical Radiophysics. New York: Springer-Verlag, 1987

24 Zheng Z H, Xuan Y M. Theory of near-field radiative heat transfer for stratified magnetic media. Int J Heat Mass Transfer, 2011, 54: 1101-1110

25 Skaar J. Fresnel equations and the refractive index of active media. Phys Rev E, 2006, 73: 026605

26 Fu C J, Zhang Z M. Thermal radiative properties of metamaterials and other nanostructured materials: A review. Front Energy Power Eng China, 2009, 3: 11-26

27 Sipe J E. New Green-function formalism for surface optics. J Opt Soc Am B, 1987, 4: 481-489

28 Cheng X C, Fu Q H, Zhao X P. Spatial separation of spectrum inside the trapered metamaterial optical waveguide. Chinese Sci Bull, 2011, 56: 209-214

29 Chakrabarti S, Ramakrishna S A, Wanare H. Coherently controlling metamaterials. Opt Express, 2008, 16: 19504-19511

30 Pendry J B, Holden A J, Stewart W J, et al. Extremely low frequency plasmons in metallic mesostructures. Phys Rev Lett, 1996, 76: 4773-4776

31 Pendry J B, Holden A J, Robbins D J, et al. Magnetism from conductors and enhanced nonlinear phenomena. IEEE Trans Microwave Theory Tech, 1999, 47: 2075-2084

32 Smith D R, Padilla W J, Vier D C, et al. Composite medium with simultaneously negative permeability and permittivity. Phys Rev Lett, 2000, 84: 4184-4187

33 Fu C J. Radiative properties of emergings materials and radiation heat transfer at the nanoscale. Doctoral Dissertation. Atlanta: Georgia Institute of Technology, 2004

34 Veselago V G. The electrodynamics of substances with simultaneously negative values of $\varepsilon$ and $\mu$. Sov Phys Usp, 1968, 10: 509-514

$35 \mathrm{Fu} \mathrm{C} \mathrm{J,} \mathrm{Zhang} \mathrm{Z} \mathrm{M.} \mathrm{Planar} \mathrm{heterogeneous} \mathrm{structures} \mathrm{for} \mathrm{coherent}$ emission of radiation. Opt Lett, 2005, 30: 1873-1875

36 Park K, Lee B J, Fu C J, et al. Study of the surface and bulk polaritons with a negative index metamaterial. J Opt Soc Am B, 2005, 22: 1016-1023

37 Francoeur M, Menguc M P, Vaillon R. Spectral tunning of near-field radiative heat flux between two thin silicon carbide films. J Phys D: Appl Phys, 2010, 43: 075501

38 Francoeur M, Menguc M P, Vaillon R. Local density of electromagnetic states within a nanometric gap formed between two thin films supporting surface phonon polaritons. J Phys D: Appl Phys, 2010, 107: 034313

39 Francoeur M, Menguc M P. Role of fluctuational electrodynamics in near-field radiative heat transfer. J Quant Spectrosc Radiat Transfer, 2008, 109: 280-293

Open Access This article is distributed under the terms of the Creative Commons Attribution License which permits any use, distribution, and reproduction in any medium, provided the original author(s) and source are credited. 